\title{
Características Psicométricas da Adaptação Portuguesa do Explora - Questionário para a Orientação de Carreira
}

\author{
Psychometric Characteristics of a Portuguese Adaptation of Explora - Questionnaire \\ for Career Guidance
}

\begin{abstract}
Alexandra Barros ${ }^{1}$
\section{Resumo}

O presente trabalho reporta a adaptação portuguesa do Explora - Questionário para a Orientação de Carreira, analisando indicadores de precisão e de validade numa amostra portuguesa de estudantes do nono ano de escolaridade de várias escolas públicas portuguesas dos concelhos de Lisboa, Setúbal e Beja (n=492), em que 244 (49.6\%) são do sexo feminino e 248 (50.4\%) do sexo masculino, com uma média etária de 14.7 anos e uma mediana de 15 anos. Os indicadores de consistência interna, a análise em componentes principais das subescalas com rotação varimax e as correlações das escalas com as de um instrumentode avaliação de interesses já validado revelam que este instrumento mantém, na adaptação portuguesa, boas características psicométricas e semelhantes às da versão original. Estes resultados permitem considerar o Explora um instrumento adequado para a utilização com estudantes desta faixa etária, podendo ajudá-los nas escolhas educacionais no fim do nono ano de escolaridade.
\end{abstract}

Palavras-chave: interesses vocacionais, inventários de interesses, escolhas vocacionais, Explora

\begin{abstract}
The present study reports the Portuguese adaptation of the Explora - Questionnaire for Career Guidance, analyzing indicators of precision and validity in a Portuguese sample of students from the 9th grade in Portuguese public schools in Lisbon, Setúbal and Beja $(n=492), 244$ (49.6\%) female and 248 (50.4\%) males, with a mean age of 14.7 years and a median of 15 years. The internal consistency indicators, the principal component analysis with varimax rotation, and the correlations with other instruments of interest assessment already adapted for Portugal, show that this instrument maintains good psychometric characteristics in the Portuguese adaptation and similar to the original version. These results allow us to consider the Explora an instrument suitable for use with students of this age group, that can help them in the educational choices at the end of the 9th year of schooling.
\end{abstract}

Keywords: vocational interests, interest inventory, vocational choices, Explora

\footnotetext{
${ }^{1}$ Faculdade de Psicologia da Universidade de Lisboa, Portugal, CICPSI. Docente e Subdiretora. Alameda da Universidade 1649-013 Lisboa, Portugal. Tel.: 00351919270789. E-mail: afbarros@psicologia.ulisboa.pt

Revista Iberoamericana de Diagnóstico y Evaluación - e Avaliação Psicológica. RIDEP · №55 · Vol.2 · 19-30 2020

ISSN: 1135-3848 print /2183-6051online
} 


\section{Introdução}

No atual contexto educativo e de trabalho, e numa perspetiva de aprendizagem e de desenvolvimento ao longo de toda a vida, com mais transições e com desafios significativamente maiores do que as gerações anteriores, os indivíduos têm que fazer várias escolhas vocacionais ao longo da sua carreira (Amundson, Mills, \& Smith, 2014; Barros, 2010; Chudzikowski, 2012; Hirschi, 2018; Van Esbroeck, 2008), num percurso individual em que o self é o integrador de um conjunto de variáveis individuais, como as necessidades, os valores ou os interesses (Super, Savickas, \& Super, 1996). Ao longo deste percurso de vida, o aconselhamento vocacional deve ajudar os indivíduos a assumirem o controlo sobre a construção da sua carreira (Savickas et al, 2009), sendo o autoconhecimento uma dimensão central para as suas tomadas de decisão. Nesse sentido, os inventários de interesses, pela forma como permitem dar ao indivíduo o acesso a dados sobre os seus interesses inventariados, contribuem significativamente para os processos de intervenção que visam o desenvolvimento humano em períodos de transição ao longo da vida (Savickas, 2013a).

Desempenhando um papel fundamental na identidade dos adolescentes e na exploração de carreira, os interesses vocacionais assumem um papel central na literatura científica e nos modelos da Psicologia Vocacional (Hansen, 2005; Sodano, 2015; Su, 2018), sendo conceptualizados como traços ou tendências disposicionais, com um carácter de estabilidade e de predisposição para se envolver num grupo especifico e homogéneo de estímulos ambientais, aumentando a prontidão para lhes prestar atenção e para agir (Rounds \& Su, 2014; Savickas, 1999; Savickas \& Spokane, 1999). Este interesse como traço distingue-se do interesse enquanto estado motivacional (Savickas, 1999; Su, Stoll, \& Rounds, 2018).

Analisando o papel dos interesses nos principais modelos da Psicologia Vocacional, é consensual a centralidade deste conceito. De acordo com a teoria sociocognitiva da carreira (SCCT) (Lent, 2013; Lent \& Brown, 2013, 2017; Lent, Brown, \& Hackett, 1994), modelo baseado no modelo sociocognitivo de Bandura (1977) os indivíduos são expostos a diferentes contextos e oportunidades de aprendizagem, recebendo reforços diferenciados quando realizam determinadas atividades. Nesta relação dinâmica entre os indivíduos, o seu comportamento e o seu ambiente, tende a haver fortalecimento do interesse por atividades em que o indivíduo se percebe como competente e face às quais antecipa resultados positivos - crenças de autoeficácia e expectativas de resultado (Lent, 2013; Lent \& Brown, 2013, 2017; Lent, Brown, \& Hackett, 1994). No modelo de Desenvolvimento de Carreira de Super $(1990,1995)$ também se refere a importância dos interesses e da sua avaliação no decorrer da adolescência e início da idade adulta para promover a exploração de si próprio e o autoconhecimento, no sentido de facilitar tomadas de decisão de carreira fundamentadas. Já para o modelo dos tipos de personalidade e de ambientes de trabalho de Holland (1973, 1985, 1997), os interesses configuram a expressão da personalidade no contexto ocupacional, pelo que os inventários de interesses podem ser vistos como inventários de personalidade que permitem posicionar os indivíduos face à sua semelhança com seis tipos de personalidade que agrupam características como valores, competências, interesses, traços e preferências (Realista, Investigativo, Artístico, Social, Empreendedor e Convencional).

Embora os interesses não sejam a única variável envolvida nas diferentes escolhas educacionais e profissionais que o indivíduo tem que fazer ao longo do seu percurso de vida (Wang \& Wanberg, 2017), havendo influência de fatores familiares, sociais, económicos, culturais (e.g., Ahn, Usher, Butz, \& Song, 2016; Flores et al., 2017; Fouad \& Santana, 2017; Gunderson, Ramirez, Levine, \& Beilock, 2012), do género (e.g., Iskander, Gore, Furse, \& Bergenson, 2013), de especificidades individuais como as perceções de competência ou os significados atribuídos aos acontecimentos de vida (e.g., Bandura et al, 2001; Lent \& Brown, 2013; Savickas, 2013b; Savickas et al., 2009; Super, Savickas, \& Super, 1996; Teixeira \& Laranjeira, 2018), ou mesmo fatores de acaso (e.g., Krumboltz, Foley, \& Cotter, 2013), os estudos revelam uma relação significativa entre os interesses, as intenções de escolha e/ou o desempenho (Falk, Rottinghaus, Casanova, 
Borgen, \& Betz, 2017; Lent et al , 2018; Nye, Su, Rounds, \& Drasgow, 2017; Sheu et al, 2010).

Através de inventários de interesses, é possível definir a força relativa dos interesses enquanto traços, ou seja, o quanto uma preferência por determinada atividade se posiciona relativamente a outros interesses, pelo que a medida dos interesses e a devolução desses dados aos próprios indivíduos favorece $\mathrm{o}$ desenvolvimento do seu autoconceito vocacional (Savickas, 1999), o que pode ajudá-los a lidar com as escolhas que vão fazendo ao longo do seu percurso escolar e profissional (Krumboltz, Foley, \& Cotter, 2013; Masdonati \& Fourier, 2015; Savickas et al., 2009; Sodano, 2011).

O sistema educativo português permite percursos diversos, mas impõe, no final do terceiro ciclo, uma tomada de decisão sobre as vias possíveis para obter a escolaridade obrigatória e sobre a área de estudos pretendida. Neste processo, a intervenção vocacional, ao nível do nono ano, assume uma função de promoção da maturidade vocacional (Super, Savickas, \& Super, 1996) trabalhando, entre outras dimensões, o autoconhecimento. É com esse objetivo que é feita a avaliação psicológica, com ênfase nas aptidões e nos interesses, de forma a devolver aos jovens informação sobre o seu próprio perfil, suscitando a reflexão, a metacognição e a autorregulação na construção dos seus projetos de vida (Abreu, 2002).

Embora haja vários métodos de recolher informações sobre os interesses (Super, 1964), os questionários de autorrelato em que $\mathrm{o}$ próprio indivíduo responde a itens sobre as suas preferências, por serem baseados em estudos de validade e de precisão e em que os resultados obtidos são posicionados face a grupos normativos, são um método fundamental para obter um perfil de interesses (Sodano, 2015). Esse perfil de interesses pode ser avaliado com diferentes graus de especificidade e a medida a partir de dimensões de largo espetro como os seis tipos RIASEC de Holland constitui um enquadramento parcimonioso muito útil para ajudar os indivíduos nessa exploração de carreira ( $\mathrm{Su}, 2018$ ).

O Explora é um instrumento de avaliação que permite caracterizar os interesses dos jovens, partilhando com o modelo dos tipos de personalidade e dos ambientes de trabalho de Holland uma perspetiva tipológica e diferencial.
Admitindo que as escolhas vocacionais são uma expressão da personalidade do indivíduo, este questionário de interesses parte de uma transformação dos resultados num perfil com base em 6 campos profissionais com correspondência aos tipos de meio de trabalho de Holland (Holland, 1973, 1985, 1997), assumindo a importância da congruência entre o tipo de personalidade e o tipo de ambiente profissional/escolar para a satisfação e estabilidade dos indivíduos. O facto do Explora considerar, para a avaliação dos campos profissionais, não só as preferências por profissões, mas também por determinadas atividades, e pelas competências e características de personalidade que o indivíduo perceciona em si próprio, permite ultrapassar uma escolha assente em estereótipos profissionais e ainda traz para a definição do perfil de interesses do indivíduo, a sua autoeficácia face a vários domínios e as características que vê em si.

$\mathrm{O}$ instrumento original foi desenvolvido em Espanha e as suas propriedades psicométricas foram obtidas a partir de uma amostra espanhola e confirmadas com amostras recolhidas no Equador, Colômbia e México (Martínez-Vicente \& Santamaría, 2013).

O presente trabalho visa fazer o estudo das características psicométricas da versão portuguesa do Explora, procurando assim contribuir para que os psicólogos portugueses disponham de um instrumento de avaliação dos interesses adaptado para amostras portuguesas.

\section{Método}

\section{Participantes}

Os participantes são 492 alunos do nono ano de escolaridade que frequentam escolas públicas dos distritos de Lisboa, de Setúbal e de Beja, sendo 140 participantes de Lisboa, 187 de Setúbal e 165 de Beja, 244 raparigas e 248 rapazes no total, com idades entre os 13 anos e os 19 anos, com mediana 15 .

\section{Instrumentos \\ EXPLORA - Questionário para a Orientação da Carreira}

O Explora é constituído por 180 itens referentes a 6 campos profissionais Técnicomanual (T), Científico-investigador (C), Artístico- 
criativo (A), Social-assistencial (S), Económicoempresarial (E) e Escritório-administrativo (O), com correspondência com os tipos RIASEC (Realista, Investigador, Artístico, Social, Empreendedor e Convencional) de Holland (Holland, 1997; Holland, Fritzsche, \& Powell, 1994). Cada um destes campos tem uma escala total e subescalas relacionadas com interesses por atividades e por profissões (secção 1: gosto ou não gosto de uma determinada atividade ou profissão), autoavaliação de competências e aptidões (secção 2: tenho ou não tenho um determinado conjunto de competências ou de aptidões), e autoavaliação de características pessoais (secção 3: considerome uma pessoa...). Os itens são de resposta nominal (Sim /?/ Não) e a sua redação tem elevada validade facial, procurando refletir as mudanças que têm caracterizado o mundo do trabalho, com uma linguagem atual, facilmente compreendida pelos destinatários.

O Explora pode ser aplicado em grupo ou individualmente, a indivíduos a partir dos 12 anos até à idade adulta.

A adaptação portuguesa do Explora foi feita a partir da tradução por um psicólogo bilingue (português-castelhano) e revista por uma equipa de psicólogos. A versão assim traduzida e revista foi, depois, aprovada pela editora da versão original - TEA-Ediciones - e pela Hogrefe, editora da versão portuguesa. $\mathrm{O}$ instrumento original foi desenvolvido em Espanha com 3570 sujeitos (45\% do sexo masculino e $55 \%$ do sexo feminino), em que 3177 são estudantes do terceiro ciclo e secundário, com média etária de 15 anos e ensino superior, com média etária de 24.7 anos e 393 são adultos não estudantes com idade média de 29.8. As boas características psicométricas da versão original do Explora traduzem-se numa boa validade facial, em que os itens são formulados a partir de afirmações adequadas às diferentes profissões e atividades características dos tempos atuais. Os valores de consistência interna obtidos nas pontuações totais de cada escala são elevados, com valores iguais ou superiores a .92, sendo .92 para as escalas totais dos campos Artísticocriativo e Económico-empresarial, .93 para as dos campos Científico-investigador e Escritórioadministrativo e .94 para os campos Técnicomanual e Social-assistencial. Estes valores permitem concluir que este questionário oferece bons níveis de precisão na interpretação do perfil global. Quando se consideram as subescalas do perfil específico, baseado em subescalas, os valores do coeficiente alfa de Cronbach são menos elevados, mas ainda satisfatórios com um coeficiente alfa de Cronbach médio de .84. O cálculo de correlações entre os itens e o total corrigido da escala a que pertence permite concluir que $81.5 \%$ dos itens têm um bom nível discriminatório, com as pontuações das correlações item-total corrigido se acima de .40, sendo que somente $5.3 \%$ apresentam correlações iguais ou inferiores a .19 (Martínez-Vicente \& Santamaría, 2013).

Ainda na versão original, na análise fatorial dos 180 itens, foram extraídos seis fatores que permitem reconhecer claramente os campos Técnico-manual (T), Científico-investigador (C), Artístico-criativo (A) e Social-assistencial (S). Embora alguns itens do campo Económicoempresarial (E) saturem no fator que corresponde ao campo Escritório-administrativo (O) nas amostras mais jovens, o facto destes fatores tenderem a ser mais diferenciados em amostras de participantes mais velhos e a referência ao modelo RIASEC que suporta este instrumento, tornam recomendável que ambos os campos continuem a ser avaliados separadamente (Martínez-Vicente \& Santamaría, 2013).

Na versão portuguesa, o Explora, tal como na versão original, permite obter, para cada indivíduo, um perfil profissional global que é representado graficamente e que indica a pontuação do avaliado em cada um dos seis campos profissionais. Um código sumário de duas letras (à semelhança dos códigos de Holland) sintetiza os campos profissionais em que as pontuações são mais elevadas e permite dirigir a procura do indivíduo, relativamente a determinados grupos profissionais e/ou ofertas escolares ou formativas relacionadas com os mesmos. Obtém-se ainda um perfil profissional específico que fornece informação diferenciada relativamente à área de interesses por atividades, à área de interesses por profissões, à área de aptidões e competências e à área das características pessoais percebidas. Este perfil específico permite analisar se o interesse do indivíduo pelas atividades e pelas profissões que lhes correspondem é homogéneo, permitindo 
também comparar o seu interesse por atividades e por profissões com a autoavaliação que faz das suas competências e aptidões em determinado campo. Os resultados obtidos também permitem verificar se existe inconsistência de respostas a itens que avaliam o mesmo interesse/capacidade ou característica, ou se o indivíduo respondeu muitas vezes na opção interrogativa (?) (MartínezVicente \& Santamaría, 2013).

\section{Self-Directed Search - SDS}

O Self-Directed Search - SDS foi traduzido para a língua portuguesa com a permissão da Psychological Assessment Resources - PAR, tendo sido estudado e validado com amostras de Portugal e Brasil (Primi, Mansão, Muniz, \& Nunes, 2010; Teixeira, Barros, \& Janeiro, 2010). É um questionário constituído por um total de 216 itens, distribuídos por 6 escalas que correspondem aos tipos $\mathrm{R}$ (Realista) I (Investigativo) A (Artístico), S (Social), E (Empreendedor) e C (Convencional), segundo o modelo RIASEC de Holland (1973, 1985, 1997). Na escala Atividades, o sujeito manifesta a sua preferência por determinadas atividades (66 itens, 11 por subescala), na escala Competências estima as suas competências e aptidões em relação a cada um dos seis tipos (66 itens, 11 por subescala) e na escala Carreiras assinala a sua preferência em relação a determinadas profissões de cada um dos seis tipos (72 itens, 12 por subescala). Estas 3 escalas são constituídas por 204 itens de resposta nominal (Sim/Não). A escala Classificando As Suas Aptidões, é constituída por dois subconjuntos, relacionados com as escalas RIASEC (MecânicaR, Científica-I, Artística-A, Para Ensinar-S, Para Vendas-E, Para Trabalho de Escritório-C, e Manual-R, Matemática-I, Musical-A, Compreender os Outros-S, Para Gestão-E e Administrativa-C) de acordo com as aptidões que pessoa pensa que tem, quando se compara com os outros da mesma idade, numa escala (Baixa/Média/Elevada) que vai de 1 (valor mais baixo) a 7 (valor mais elevado).

Os valores de consistência interna, avaliados através do coeficiente de alfa de Cronbach nas amostras portuguesas, foram iguais ou superiores a $.91 \mathrm{e} \mathrm{a}$ análise fatorial aos itens permite reconhecer a estrutura em 6 fatores do instrumento, apontando para as boas características psicométricas desta versão do SDS (Primi et al, 2010, Teixeira et al., 2010).

\section{Procedimento}

O presente estudo foi aprovado pela Comissão Especializada de Deontologia da Faculdade de Psicologia da Universidade de Lisboa certificando que cumpre todos os princípios deontológicos e éticos definidos pela Ordem dos Psicólogos Portugueses. Foram, assim, garantidos a confidencialidade, o anonimato dos dados recolhidos e o caráter voluntário da participação, tendo sido obtido o consentimento informado. Como os participantes eram menores foi necessária a aprovação, pelos encarregados de educação, da participação dos seus educandos na investigação. No cumprimento das exigências legais, foi dirigido o formulário de pedido de Monitorização de Instrumento em Meio Escolar (MIME) à Direção Geral de Educação e foram pedidas autorizações às Direções dos Agrupamentos de Escolas.

Foram calculados os coeficientes alfa de Cronbach das escalas e subescalas do Explora, correlações de cada item com o total da escala corrigido (excluindo o próprio item), cálculo do efeito da omissão de cada item no coeficiente alfa de Cronbach de cada escala, análise fatorial das subescalas, e correlações entre as escalas e subescalas do Explora e as do SDS: Self-Directed Search (Primi, Mansão, Muniz, \& Nunes, 2010) A recolha de dados foi realizada presencialmente, em formato papel e os dados recolhidos foram analisados com recurso ao programa IBM SPSS Statistics Version 25.

\section{Resultados}

\section{Características Psicométricas da versão portuguesa do Explora}

Para analisar a fiabilidade do Explora, de acordo com o critério da consistência interna, recorreu-se ao cálculo do coeficiente alfa de Cronbach das diferentes escalas e subescalas, bem como da correlação de cada item com o total da escala a que pertence (excluindo o próprio item) (Quadros 1 a 3).

Os coeficientes alfa de Cronbach referentes às escalas totais variam entre um mínimo de .91 para 
Quadro 1. Indicadores de Consistência Interna das Escalas Técnico-Manual e Científico-Investigador

\begin{tabular}{|c|c|c|c|c|c|c|}
\hline \multirow[b]{2}{*}{ Subescalas } & \multicolumn{3}{|c|}{$\begin{array}{l}\text { Escala Técnico-Manual: coeficiente alfa de } \\
\text { Cronbach } .93(\mathrm{~N}=485)\end{array}$} & \multicolumn{3}{|c|}{$\begin{array}{l}\text { Escala Científico-investigador: coeficiente alfa de } \\
\text { Cronbach .94 }(\mathrm{N}=486)\end{array}$} \\
\hline & $\mathrm{N}^{\mathrm{o}}$ Itens & $\begin{array}{l}\text { Correlação de ite } \\
\text { total corrigido } \\
\text { (mínimo e } \\
\text { máximo) }\end{array}$ & $\begin{array}{l}\text { Coeficiente alfa de } \\
\text { Cronbach }\end{array}$ & $\mathrm{N}^{\circ}$ Itens & $\begin{array}{l}\text { Correlação de iten } \\
\text { total corrigido } \\
\text { (mínimo e } \\
\text { máximo) }\end{array}$ & $\begin{array}{l}\text { Coeficiente alfa de } \\
\text { Cronbach }\end{array}$ \\
\hline Profissões & 9 & $.33-.59$ & .77 & 9 & $.39-.62$ & .81 \\
\hline Atividades & 9 & $.37-.62$ & .80 & 9 & $.55-.72$ & .88 \\
\hline Competências & 9 & $.47-.73$ & .88 & 9 & $.53-.72$ & .85 \\
\hline $\begin{array}{l}\text { Características } \\
\text { Pessoais }\end{array}$ & 3 & $.30-.52$ & .63 & 3 & $.49-.56$ & .70 \\
\hline
\end{tabular}

Quadro 2. Indicadores de Consistência Interna das Escalas Artístico-criativo e Social-assistencial

\begin{tabular}{|c|c|c|c|c|c|c|}
\hline & \multicolumn{3}{|c|}{$\begin{array}{c}\text { Escala Artístico-criativo: coeficiente alfa de } \\
\text { Cronbach .92 }(\mathrm{N}=484)\end{array}$} & \multicolumn{3}{|c|}{$\begin{array}{l}\text { Escala Social-assistencial: coeficiente alfa de } \\
\text { Cronbach } .93(\mathrm{~N}=484)\end{array}$} \\
\hline Subescalas & $\begin{array}{l}\mathrm{N}^{\mathrm{o}} \\
\text { Itens }\end{array}$ & $\begin{array}{l}\text { Correlação de } \\
\text { item total } \\
\text { corrigido } \\
\text { (mínimo e } \\
\text { máximo) }\end{array}$ & $\begin{array}{l}\text { Coeficiente alfa } \\
\text { de Cronbach }\end{array}$ & $\mathrm{N}^{\mathrm{o}}$ Itens & $\begin{array}{l}\text { Correlação de } \\
\text { item total } \\
\text { corrigido } \\
\text { (mínimo e } \\
\text { máximo) }\end{array}$ & $\begin{array}{l}\text { Coeficiente alfa } \\
\text { de Cronbach }\end{array}$ \\
\hline Profissões & 9 & $.23-.55$ & .74 & 9 & $.36-.62$ & .81 \\
\hline Atividades & 9 & $.40-.58$ & .78 & 9 & $.40-.71$ & .85 \\
\hline Competências & 9 & $.44-.70$ & .82 & 9 & $.38-.69$ & .83 \\
\hline $\begin{array}{l}\text { Caracteristicas } \\
\text { Pessoais }\end{array}$ & 3 & $.56-.59$ & .75 & 3 & $.51-.58$ & .73 \\
\hline
\end{tabular}

Quadro 3. Indicadores de Consistência Interna das Escalas Empresarial-persuasivo e Escala Escritório-administrativo

\begin{tabular}{|c|c|c|c|c|c|c|}
\hline \multirow[b]{2}{*}{ Subescalas } & \multicolumn{3}{|c|}{$\begin{array}{c}\text { Escala Empresarial-persuasivo: coeficiente } \\
\text { alfa de Cronbach .94 }(\mathrm{N}=483)\end{array}$} & \multicolumn{3}{|c|}{$\begin{array}{l}\text { Escala Escritório-administrativo: coeficiente } \\
\text { alfa de Cronbach } .91(\mathrm{~N}=482)\end{array}$} \\
\hline & $\begin{array}{l}\mathrm{N}^{\mathrm{o}} \\
\text { Itens }\end{array}$ & $\begin{array}{l}\text { Correlação } \\
\text { de item total } \\
\text { corrigido } \\
\text { (mínimo e } \\
\text { máximo) }\end{array}$ & $\begin{array}{l}\text { Coeficiente alfa } \\
\text { de Cronbach }\end{array}$ & $\mathrm{N}^{\mathrm{o}}$ Itens & $\begin{array}{l}\text { Correlação de } \\
\text { item total } \\
\text { corrigido } \\
\text { (mínimo e } \\
\text { máximo) }\end{array}$ & $\begin{array}{l}\text { Coeficiente alfa } \\
\text { de Cronbach }\end{array}$ \\
\hline Profissões & 9 & $.42-.65$ & .83 & 9 & $.35-.61$ & .80 \\
\hline Atividades & 9 & $.45-.69$ & .85 & 9 & $.39-.65$ & .81 \\
\hline Competências & 9 & $.50-.66$ & .87 & 9 & $.39-.62$ & .80 \\
\hline $\begin{array}{l}\text { Caracteristicas } \\
\text { Pessoais }\end{array}$ & 3 & $.41-.54$ & .67 & 3 & $.44-.55$ & .68 \\
\hline
\end{tabular}

a escala Escritório-administrativo e de .94 para as escalas Científico-investigador e Empresarialpersuasivo, valores que se podem considerar muito elevados. Quando se consideram as subescalas Profissões e de Atividades, os valores mínimos do coeficiente alfa de Cronbach são referentes ao campo Artístico-criativo (.74 para Profissões e .78 para Atividades) e os máximos referentes ao campo Empresarial-persuasivo para Profissões (.83) e Científico-investigador para Atividades (.88). Nas subescalas de Competências, todos os valores do coeficiente alfa de Cronbach são iguais ou superiores a .80 , com um máximo de .88 para o campo Técnico-manual. Os coeficientes alfa de Cronbach referentes às escalas de Características Pessoais são os mais baixos em todas as escalas, com um mínimo de .63 para a escala Técnicomanual e um máximo de .75 para a esala Artísticocriativo, o que deverá estar relacionado com o facto de serem escalas com um reduzido número de itens. A análise das correlações dos itens com as escalas a que pertencem, que variam entre moderadas a fortes, e o impacto positivo de cada item no coeficiente alfa de Cronbach de cada escala, contribuem para apoiar a consistência interna do Explora.

Com base nos resultados obtidos, pode considerar-se que a versão portuguesa do Explora possui muito boa consistência interna, com bons indicadores de fiabilidade nas escalas totais e nas subescalas Profissões e Atividades e de Competências/aptidões. 
Quadro 4. Análise em Componentes Principais das Subescalas com Rotação Varimax

\begin{tabular}{|c|c|c|c|c|c|c|}
\hline & Fator 1 & Fator 2 & Fator 3 & Fator 4 & Fator 5 & Fator 6 \\
\hline Profissões Técnico-Manual & .26 & .15 & .09 & .83 & .03 & -.21 \\
\hline AtividadesTécnico-Manual & .14 & .08 & .09 & .90 & -.08 & -.10 \\
\hline CompetênciasTécnico-Manual & .10 & .26 & .14 & .78 & -.02 & .33 \\
\hline Caract. PessoaisTécnico-Manual & .09 & 17 & -.02 & .73 & -.09 & .35 \\
\hline Profissões Científico-investigador & .14 & .85 & .14 & .13 & .18 & -.10 \\
\hline Atividades Científico-investigador & .13 & .90 & .12 & .24 & .08 & -.01 \\
\hline Competências Científico-investigador & .05 & .84 & .16 & .21 & .07 & .30 \\
\hline Caract. Pessoais Científico-investigador & .01 & .70 & .16 & .06 & .19 & .41 \\
\hline Profissões Artístico-criativo & .18 & .12 & .85 & .15 & .18 & -.11 \\
\hline Atividades Artístico-criativo & .13 & .20 & .82 & 11 & .22 & -.08 \\
\hline Competências Artístico-criativo & .06 & .11 & .82 & .00 & .30 & .17 \\
\hline Caract. Pessoais Artístico-criativo & .04 & .11 & .74 & .05 & .08 & .37 \\
\hline Profissões Social-Assistencial & .17 & .06 & .22 & .03 & .81 & -.17 \\
\hline Atividades Social-Assistencial & .15 & .13 & .23 & -.07 & .86 & -.04 \\
\hline Competências Social-Assistencial & .06 & .27 & .19 & -.09 & .80 & .27 \\
\hline Caract. Pessoais Social-Assistencial & .13 & .05 & .14 & -.05 & .69 & .38 \\
\hline Profissões Empresarial-persuasivo & .90 & .01 & .14 & .09 & .10 & .08 \\
\hline Atividades Empresarial-persuasivo & .83 & .09 & .20 & .10 & .08 & .16 \\
\hline Competências Empresarial-persuasivo & .62 & .20 & .29 & .07 & .16 & .50 \\
\hline Caract. Pessoais Empresarial-persuasivo & .53 & .11 & .17 & .02 & .06 & .55 \\
\hline Profissões Escritório-administrativo & .85 & .00 & .02 & .08 & .19 & -.05 \\
\hline Atividades Escritório-administrativo & .86 & .07 & -.04 & .20 & .03 & .02 \\
\hline Competências Escritório-administrativo & .58 & .22 & .05 & .20 & .06 & .48 \\
\hline Caract. Pessoais Escritório-administrativo & .43 & .24 & .01 & .15 & .19 & .47 \\
\hline Valor próprio & 8.06 & 3.29 & 2.85 & 1.84 & 1.41 & 1.09 \\
\hline variância & 33.6 & 13.7 & 11.86 & 7.67 & 5.9 & 4.53 \\
\hline
\end{tabular}

As saturações mais elevadas em cada fator encontram-se a negrito.

Quadro 5. Correlações de Pearson entre os totais do Explora e os totais do SDS

\begin{tabular}{|c|c|c|c|c|c|c|}
\hline SDS/Explora & $\begin{array}{l}\text { Técnico/ } \\
\text { manual }\end{array}$ & $\begin{array}{l}\text { Científico/ } \\
\text { investigador }\end{array}$ & $\begin{array}{l}\text { Artistico/ } \\
\text { criativo }\end{array}$ & $\begin{array}{c}\text { Social/ } \\
\text { assistencial }\end{array}$ & $\begin{array}{c}\text { Empresarial/ } \\
\text { persuasivo }\end{array}$ & $\begin{array}{c}\text { Escritório/ } \\
\text { administrativo }\end{array}$ \\
\hline $\mathrm{R}$ & $.64 * *$ & $.24 * *$ & $.18 *$ & -.03 & .11 & .12 \\
\hline I & $.20 * *$ & $.75 * *$ & $.29 * *$ & $.26 * *$ & .14 & $.18^{*}$ \\
\hline A & $.19 *$ & $.39 * *$ & $.71 * *$ & $.39 * *$ & $.20 * *$ & .13 \\
\hline S & .06 & $.34 * *$ & $.42 * *$ & $.68 * *$ & $.28 * *$ & $.22 * *$ \\
\hline E & $.19 *$ & $.25 * *$ & $.20 * *$ & $.19 *$ & $.75^{* *}$ & $.57 * *$ \\
\hline $\mathrm{C}$ & .12 & $.25^{* *}$ & .10 & $.22 * *$ & $.55^{* *}$ & $.60 * *$ \\
\hline
\end{tabular}

* Significativo a $p<.05 * *$ Significativo a $p<.01$

As subescalas Características Pessoais, isoladamente, apresentam uma consistência interna menos satisfatória, embora aceitável, tal como acontece na versão original. Sendo constituídas por apenas 3 itens, não devem ser interpretadas isoladamente.

Uma análise em componentes principais das subescalas revela 6 fatores com valor próprio superior a 1 que, no seu conjunto, justificam 77.26 \% da variância total dos dados. O Quadro 4 apresenta a matriz, após rotação varimax, onde se encontram os valores com maior saturação em cada fator.

Como se pode verificar no Quadro 4, o fator 1 e o fator 6 parecem ter uma correspondência com as escalas Empresarial-persuasivo e Escritórioadministrativo, o fator 2 com a escala Científicoinvestigador, o fator $3 \mathrm{com}$ a escala Artísticocriativo, o fator 4 com a escala Técnico-Manual, e o fator 5 com a escala Social-Assistencial. Esta estrutura, embora não confirme inteiramente a estrutura em 6 fatores do instrumento, é indicadora da validade de construção do instrumento e está de acordo com os resultados obtidos na versão original.

No sentido de obter mais indicadores de validade, e uma vez que a construção do Explora se baseia no modelo de Holland, admitindo a correspondência entre os 6 campos profissionais com os 6 tipos RIASEC, os resultados das Escalas totais do Explora foram correlacionados com os resultados com a adaptação do Self-Directed Search (Primi, Mansão, Muniz, \& Nunes, 2010) (Quadro 5).

As correlações mais elevadas do total de cada escala do Explora referem-se sempre ao tipo correspondente no SDS, sendo a correlação da escala Técnico-manual com o tipo Realista de .64, a 
Quadro 6. Correlações de Pearson entre as subescalas Atividades do Explora e as do SDS

\begin{tabular}{lcccccc}
\hline $\begin{array}{l}\text { SDS/ } \\
\text { EXPLORA }\end{array}$ & $\begin{array}{c}\text { Técnico/ } \\
\text { manual }\end{array}$ & $\begin{array}{c}\text { Científico/ } \\
\text { investigador }\end{array}$ & $\begin{array}{c}\text { Artistico/ } \\
\text { criativo }\end{array}$ & $\begin{array}{c}\text { Social/ } \\
\text { assistencial }\end{array}$ & $\begin{array}{c}\text { Empresarial/ } \\
\text { persuasivo }\end{array}$ & $\begin{array}{c}\text { Escritório/ } \\
\text { administrativo }\end{array}$ \\
\hline $\mathrm{R}$ & $\mathbf{. 5 3} * *$ & .02 & .03 & -.13 & .01 & .02 \\
$\mathrm{I}$ & .07 & $\mathbf{. 6 1} * *$ & $.32^{* *}$ & $.20^{* *}$ & .10 & .07 \\
$\mathrm{~A}$ & .10 & $.27^{* *}$ & $\mathbf{. 6 1} * *$ & $.32^{* *}$ & .07 & -.01 \\
$\mathrm{~S}$ & .03 & $.23^{* *}$ & $.34^{* *}$ & $\mathbf{. 6 2} * *$ & $.19^{*}$ & $.40^{* *}$ \\
$\mathrm{E}$ & .14 & $.17^{*}$ & .14 & $.19 *$ & $\mathbf{. 6 4} * *$ & $.43^{* *}$ \\
$\mathrm{C}$ & .07 & $.19^{*}$ & -.05 & .14 & $.39 * *$ & $\mathbf{. 4 2} * *$ \\
\hline
\end{tabular}

* Significativo a $p<.05 \quad * *$ Significativo a $p<.01$

Quadro 7. Correlações de Pearson entre as subescalas Competências do Explora e as do SDS

\begin{tabular}{|c|c|c|c|c|c|c|}
\hline $\begin{array}{l}\text { SDS/ } \\
\text { EXPLORA }\end{array}$ & $\begin{array}{l}\text { Técnico/ } \\
\text { manual }\end{array}$ & $\begin{array}{l}\text { Científico/ } \\
\text { investigador }\end{array}$ & $\begin{array}{l}\text { Artistico/ } \\
\text { criativo }\end{array}$ & $\begin{array}{c}\text { Social/ } \\
\text { assistencial }\end{array}$ & $\begin{array}{c}\text { Empresarial/ } \\
\text { persuasivo }\end{array}$ & $\begin{array}{c}\text { Escritório/ } \\
\text { administrativo }\end{array}$ \\
\hline $\mathrm{R}$ & $.60 * *$ & $.35^{* *}$ & $.26 * *$ & .12 & $.22 * *$ & $.25 * *$ \\
\hline I & $.32 * *$ & $.65^{* *}$ & $.20 * *$ & $.28 * *$ & $.24 * *$ & $.25 * *$ \\
\hline A & $.28 * *$ & $.35^{* *}$ & $.62 * *$ & $.43 * *$ & $.31 * *$ & $.19 *$ \\
\hline S & $.20 * *$ & $.31 * *$ & $.41 * *$ & $.56 * *$ & $.31 * *$ & $.20 * *$ \\
\hline $\mathrm{E}$ & $.22 * *$ & $.26^{* *}$ & $.15^{*}$ & $.18^{*}$ & $.60 * *$ & $.42 * *$ \\
\hline $\mathrm{C}$ & $.23 * *$ & $.22 * *$ & $.24 * *$ & $.26 * *$ & $.38 * *$ & $.44 * *$ \\
\hline
\end{tabular}

Quadro 8. Correlações de Pearson entre as subescalas Profissões do Explora e as do SDS

\begin{tabular}{|c|c|c|c|c|c|c|}
\hline $\begin{array}{l}\text { SDS/ } \\
\text { EXPLORA }\end{array}$ & $\begin{array}{c}\text { Técnico/ } \\
\text { manual }\end{array}$ & $\begin{array}{l}\text { Científico/ } \\
\text { investigador }\end{array}$ & $\begin{array}{l}\text { Artistico/ } \\
\text { criativo }\end{array}$ & $\begin{array}{c}\text { Social/ } \\
\text { assistencial }\end{array}$ & $\begin{array}{c}\text { Empresarial/ } \\
\text { persuasivo }\end{array}$ & $\begin{array}{c}\text { Escritório/ } \\
\text { administrativo }\end{array}$ \\
\hline $\mathrm{R}$ & $.53 * *$ & $.15^{*}$ & .14 & .01 & .08 & .05 \\
\hline I & .05 & $.61 * *$ & $.16^{*}$ & $.20 * *$ & .04 & .07 \\
\hline A & .11 & $.27 * *$ & $.53 * *$ & $.44 * *$ & .11 & -.01 \\
\hline S & .04 & $.33^{* *}$ & $.29 * *$ & $.61 * *$ & $.18 *$ & .04 \\
\hline $\mathrm{E}$ & $.15^{*}$ & $.18 * *$ & $.22 * *$ & .13 & $.63 * *$ & $.54 * *$ \\
\hline $\mathrm{C}$ & .05 & .07 & -.01 & .04 & $.54 * *$ & $.49 * *$ \\
\hline
\end{tabular}

* Significativo a $p<.05 \quad * *$ Significativo a $p<.01$

da escala Científico-investigador com o tipo Investigativo de .75, a da escala Artístico-criativo com o tipo Artístico de .71, a da escala Socialassistencial com o tipo Social de .68, a da escala Empresarial-persuasivo com o tipo Empreendedor de .75 e a da escala Escritório-administrativo com o tipo Convencional de 60 .

Para obtenção de mais indicadores de validade, foram ainda calculadas as correlações entre as subescalas Atividades do Explora e do SDS (Quadro 6), as subescalas Competências Explora e do SDS (Quadro 7) e as subescalas Profissões do Explora e do SDS (Quadro 8).

Também no caso das subescalas (Atividades, Competências e Profissões), as correlações mais elevadas do total de cada subescala do Explora referem-se ao tipo correspondente no SDS, sendo a correlação da escala Técnico-manual com o tipo Realista de .53, para Atividades e para Profissões, .60 para Competências, a da escala Científicoinvestigador com o tipo Investigativo de .61 para Atividades e para Profissões e .65 para Competências, a da escala Artístico-criativo com o tipo Artístico de .61 para Atividades, .62 para Competências e .53 para Profissões, a da escala Social-assistencial com o tipo Social de .62 para Atividades, .56 para Competências e .61 para Profissões, a da escala Empresarial-persuasivo com o tipo Empreendedor de .64 para Atividades, .60 para Competências e .63 para Profissões e a da escala Escritório-administrativo com o tipo Convencional de .42 para Atividades, .44 para Competências e .49 para Profissões. No caso da escala Escritório/Administrativo do Explora nas subescalas Atividades e Profissões as correlações encontradas são ligeiramente mais elevadas com as subescalas correspondentes do tipo Empreendedor do que do tipo Convencional.

\section{Discussão}

O presente estudo revela bons indicadores de validade e de precisão da versão portuguesa do Explora, junto desta população, indicando que este questionário pode ser um instrumento muito interessante para a avaliação e a intervenção 
vocacional com alunos portugueses do nono ano de escolaridade.

Com a presente amostra, os valores de coeficientes alfa de Cronbach situam-se entre .91 (Escala Escritório-administrativo) e .94 (Escalas Científico-investigador e Empresarial-Persuasivo) para os totais das escalas dos seis campos profissionais, entre .74 (Escala Artistico-criativo) e .83 (Escala Empresarial-persuasivo) para as subescalas interesses por Profissões, entre .78 (Escala Artistico-criativo) e $\quad .88$ (Escala Científico-investigador) para as subescalas interesses por Atividades, entre .80 (Escala Escritório-administrativo) e .88 (Escala Técnicomanual) para as subescalas Competências/aptidões e entre .63 (Escala Técnico-manual) e .75 (Escala Artistico-criativo) para as subescalas Características pessoais indicando que o instrumento mantém muito boa consistência interna. Apesar de apresentar uma boa consistência interna, as subescalas Características pessoais, isoladamente, apresentam uma consistência menos satisfatória, pelo que não devem ser interpretadas separadamente. No geral, os resultados obtidos para os coeficientes alfa de Cronbach das escalas totais com a amostra portuguesa são semelhantes aos obtidos com a amostra de Espanha que variam entre .92 e .94, com os obtidos com as amostras do Equador e da Colômbia que variam entre $.90 \mathrm{e}$ .93 e com os obtidos com a amostra do México que variam entre .89 e .92 (Martínez-Vicente \& Santamaría, 2013; Martínez-Vicente \& Segura, 2014). Também ao nível das subescalas, os coeficientes alfa de Cronbach obtidos no presente estudo são semelhantes aos obtidos nas amostras de Espanha, Equador, Colômbia e México, mantendo-se a tendência para valores inferiores nas subescalas Características Pessoais (MartínezVicente \& Santamaría, 2013).

$\mathrm{Na}$ amostra portuguesa, todos os itens têm correlações com o total da escala a que pertencem, excluindo o próprio item, entre .30 e .72 , com uma única exceção em que 1 item apresenta uma correlação item-total de .23. Também nas amostras de Espanha, Colômbia, México e Equador se verifica o poder discriminativo de todos os itens, com $95 \%$ de itens com correlações com o total acima de .20 e $78 \%$ acima de .40 (Martínez-Vicente \& Santamaría, 2013).
No presente estudo, uma análise em componentes principais das subescalas, permite identificar seis fatores que justificam uma variância total dos dados de 77.26 \% . Da matriz resultante da rotação varimax, a partir das saturações das subescalas em cada fator, concluise que o fator 1 e o fator 6 parecem ter uma correspondência com os campos profissionais Empresarial-persuasivo e Escritórioadministrativo, o fator 2 com o campo profissional Científico-investigador, o fator 3 com o campo profissional Artístico-criativo, o fator 4 com o campo profissional Técnico-manual e o fator 5 com o campo profissional Social-assistencial. As saturações mais baixas referem-se às subescalas de Características pessoais. Esta estrutura, embora não confirme inteiramente a estrutura em 6 fatores do instrumento, é indicadora da validade de construção do instrumento, sendo semelhante à encontrada no estudo da versão original do Explora. A junção das escalas Empresarialpersuasivo e Escritório-administrativo no mesmo fator reproduz a estreita relação entre os tipos Empreendedor e Convencional de Holland, reportada em vários estudos (Barros, 2015; Holland, Fritzsche, \& Powell, 1994; MartínezVicente \& Valls, 2006). A análise fatorial dos itens não foi realizada na amostra portuguesa porque a relação entre o elevado número de itens e a dimensão da amostra desaconselhava a sua concretização, o que constitui uma limitação ao estudo da validade da versão portuguesa. Apesar dos bons indicadores de validade e de precisão da versão portuguesa, serão recomendáveis novos estudos com amostras mais amplas e representativas da população portuguesa que permitam generalizar os resultados.

Numa época em que a investigação sobre os interesses voltou a ter a atenção dos investigadores, e sendo saliente a relação dos interesses com as intenções de escolha académica/de carreira (Su, 2018), o desenvolvimento e a adaptação de instrumentos de avaliação dos interesses corresponde a uma necessidade dos psicólogos portugueses para a intervenção vocacional. O Sistema Educativo português impõe uma primeira opção no nono ano de escolaridade face à atual diversidade de percursos educativos disponíveis e neste nível de escolaridade, os jovens estão no estádio de 
Exploração tendo que formular preferências vocacionais gerais a partir do conhecimento de si próprios e da clarificação de interesses e valores pessoais (Super, Savickas, \& Super,1996). Por outro lado, as profundas mudanças que se têm verificado no mundo do trabalho, com o desaparecimento de muitas profissões e a emergência de outras (Hirschi, 2018) tornam necessário que a medida de interesses seja realizada a partir de inventários cujos itens sejam formulados numa linguagem atual, que represente as atividades e o contexto profissional característicos dos dias de hoje. Nesse sentido, o inventário de interesses Explora pode ser um importante instrumento para que os psicólogos portugueses possam ajudar os jovens no seu processo de autoconhecimento e na construção de intenções de escolhas educativas para o fim do nono ano de escolaridade.

\section{Referências}

Abreu, M. V. (2002). A complexidade bio-psicosócio-axiológica da personalidade humana: Contributos para uma teoria integradora. Psychologica, 30, 41-55.

Ahn, H. S., Usher, E. L., Butz, A., \& Song, M. (2016). Cultural differences in the understanding of modelling and feedback as sources of self-efficacy information. British Journal of Educational Psychology, 86(1), 112-136. doi:10.1111/bjep.12093

Amundson, N., Mills, L., \& Smith, B. (2014). Incorporating chaos and paradox into career development. Australian Journal of Career Development, 23(1), 13-21.

Bandura, A. (1977). Self-efficacy: The exercise of control. New York: W.H. Freeman and Company.

Bandura, A., Barbaranelli, C., Caprara, G. V., \& Pastorelli, C. (2001). Self-efficacy beliefs as shapers of children's aspirations and career trajectories. Child Development Journal, 72(1), 187-206.

Barros, A. (2010). Desafios da psicologia vocacional: Modelos e intervenções na era da incerteza. Revista Brasileira de Orientação Profissional, 11 (2), 165-175.

Barros, A. (2015). Contributos para a validação do Questionário de Busca Autodirigida (versão brasileira do SDS- Self-Directed Search) em estudantes cabo-verdianos. Revista Iberoamericana de Diagnóstico y Evaluación - e Avaliação Psicológica, 40(1), 62-72.

Chudzikowski, K. (2012). Career transitions and career success in the "new" career era. Journal of Vocational Behavior, 81, 298-306. doi:10.1016/j.jvb.2011.10.005

Falk, N. A., Rottinghaus, P. J., Casanova, T. N., Borgen, F. H., \& Betz, N. E. (2017). Expanding women's participation in STEM: Insignts from parallel measures of self-efficacy and interests. Journal of Career Assessment, 25(4). doi.org/10.1177/1069072716665822.

Flores, L. Y., Navarro, R. L., \& Rasheed, S. (2017). The state of SCCT research in relation to social class: Future directions. Journal of Career Assessment, 25(1), 6-23. doi:10.1177/1069072716658649.

Fouad, N., \& Santana, M. C. (2017). SCCT and underrepresented populations in STEM fields: Moving the needle. Journal of Career Assessment, 25(1) 24-39. doi:10.1177/1069072716658324

Gunderson, E. A., Ramirez, G., Levine, S. C., \& Beilock, S. I. (2012). The role of parents and teachers in the development of gender-related math attitudes. Sex Roles, 66, 156-166. doi:10.1007/s11199-011-9996-2

Krumboltz, J. D., Foley, P. F., \& Cotter, E. W. (2013). Applying the happenstance learning theory to involuntary career transitions. The Career Development Quarterly, 61, 15-26. doi:10.1002/j.2161-0045.2013.00032.x

Hansen, J. C. (2005). Assessment of interests. In S. D. Brown, \& R. W. Lent (Eds.), Career development and counseling: Putting theory and research to work (pp. 281-304). New York: Wiley.

Hirschi, A. (2018). The Fourth Industrial Revolution: Issues and Implications for career research and practice. The Career Development Quartely, 66, 192-204. doi:10.1002/cdq.12142.

Holland, J. L. (1973). Making vocational choices: A theory of careers. Englewood Cliffs, New York, NY: Prentice-Hall.

Holland, J. L. (1985). Making vocational choices. A theory of vocational personalities and work 
environments (2nd ed.). Englewood Cliffs, New Jersey: Prentice-Hall.

Holland, J. L. (1997). Making vocational choices: A theory of vocational personalities and work environments (3rd ed.). Odessa, FL: Psychological Assessment Resources.

Holland, J. L., Fritzsche, B. A., \& Powell, A. B. (1994). Technical manual for the SelfDirected. Odessa, FL: Psychological Assessment Resources.

Iskander, E. T., Gore, P. A. Jr., Furse, C., \& Bergenson (2013). Gender differences in expressed interests in engineering-related fields ACT 30-year data analysis identified trends and suggested avenues to reverse trends. Journal of Career Assessment, 21(4) 599-613. doi:10.1177/1069072712475290

Lent, R. W. (2013). Social cognitive career theory. In S.D. Brown \& R. W. Lent, (Eds.), Career development and counseling: Putting theory and research to work (2nd ed., pp. 115-146). Hoboken, NJ: Wiley

Lent, R. W., \& Brown, S. D. (2013). Social cognitive model of career self-management: Toward a unifying view of adaptive career behavior across the life span. Journal of Counseling Psychology, 60(4), 557-568.

Lent, R. W., \& Brown, S. D. (2017). Social cognitive career theory in a diverse world: Guest editors' introduction. Journal of Career Assessment, 3(1), 3-5. doi:10.1177/10690727 166.

Lent, R. W., Brown, S. D., \& Hackett, G. (1994). Toward a unifying social cognitive theory of career and academic interest, choice, and performance. Journal of Vocational Behavior, 45, 79-122.

Lent, R. W., Sheu, H. B., Miller, M. J., Cusick, M. E., Penn, L. T., \& Truong, N. N. (2018). Predictors of science, technology, engineering, and mathematics choice options: A meta-analytic path analysis of the socialcognitive choice model by gender and race/ethnicity. Journal of Counseling Psychology, 65(1), 17-35.

Masdonati, J., \& Fournier, G. (2015). Life design, young adults, and the school-to-work transition. In L. Nota \& J. Rossier (Eds.), Handbook of life design: From practice to theory and from theory to practice (pp. 117-133). Boston, MA: Hogrefe.

Martínez-Vicente, J. M., \& Santamaría, P. (2013). EXPLORA - Cuestionario para la orientación vocacional y profesional, Manual. Madrid: TEA Ediciones.

Martínez-Vicente, J. M., \& Segura, I. M. A. (2014). Características de los interesses vocacionales en alunos de educación secundaria evaluados a través del EXPLORA. International Journal of Developmental and Educational Psychology, 3(1), 549-561.

Martínez-Vicente, J. M., \& Valls, F. (2006). La elección vocacional y la planificación de la carrera. Adaptación española del SelfDirected Search (SDS-R) de Holland. Psicothema, 18(1), 117-122.

Masdonati, J., \& Fournier, G. (2015). Life design, young adults, and the school-to-work transition. In L. Nota \& J. Rossier (Eds.), Handbook of life design: From practice to theory and from theory to practice (pp. 117-133). Boston, MA: Hogrefe.

Nye, C. D., Su, R., Rounds, J., \& Drasgow, F. (2017). Interest congruence and performance: Revisiting recent metaanalytic findings. Journal of Vocational Behavior, 98, 138-151.

Primi, R., Mansão, C., Muniz, M., \& Nunes, M. (2010). SDS - Questionário de Busca Autodirigida (John Holland \& Any Powell). Manual técnico da versão brasileira. São Paulo: Casa do Psicólogo.

Rounds, J., \& Su, R. (2014). The nature and power of interests. Current Directions in Psychological Science, 23(2), 98-103.

Savickas, M. L., \& Spokane, A. R. (1999). Using vocational interests in career intervention. In M. L. Savickas \& A. R. Spokane (Eds.), Vocational interests: Meaning, measurement and counseling use (pp.253-256). Palo Alto, CA: Davies - Black.

Savickas, M. L. (1999). The psychology of interests. In M. L. Savickas \& A. R. Spokane (Eds.), Vocational interests: meaning, measurement and counseling use. (pp.19-56). Palo Alto, CA: Davies - Black

Savickas, M. L. (2013a). Ten Ideas that Changed Career Development. In M. L. Savickas (Ed.), Ten Ideas that Changed Career Development 
(pp. 1-3). Broken Arrow, OK: National Career Development Association.

Savickas, M. L. (2013b). Career construction theory and practice. In D. Brown and R. W. Lent (Eds), Career development and counseling: Putting theory and research to work (2nd ed, pp.147-183). Hoboken, NJ: John Wiley and Sons.

Savickas, M. L., Nota, L., Rossier, J., Dauwalder, J-P., Duarte, M. E., Guichard, J., et al. (2009). Life designing: A paradigm for career construction in the 21st century. Journal of Vocational Behavior, 75, 239-250. doi:10.1016/j.jvb.2009.04.004

Sheu, H. B., Lent, R. W., Brown, S. D., Miller, M. J., Hennessy, K. D., \& Duffy, R. D. (2010). Testing the choice model of social cognitive career theory across Holland themes: A metaanalytic path analysis. Journal of Vocational Behavior, 76 (2), 252-264.

Sodano, S. M. (2011). Integrating vocational interests, competencies, and interpersonal dispositions in middle school children. Journal of Vocational behavior, 79, 110-120.

Sodano, S. M. (2015). Meaning, measurement, and assessment of vocational interests for career intervention. In P. J. Hartung, M. L. Savickas, \& W. B. Walsh (Eds.), APA Handbook of Career Intervention: Volume 1. Foundations (pp. 281-301). Washington, DC: American Psychological Association.

Su, R. (2018). The three faces of interests: An integrative review of interest research in vocational, organizational, and educational psychology. Journal of Vocational Psychology. doi:doi.org/10.1016.j.jvb.2018.10.016

Su, R., Stoll, G., \& Rounds, J. (2018). The nature of interests: Toward a unifying theory of Trait-State Interest Dynamics. In C. D. Nye, \& J. Rounds (Eds.), Vocational interests: Rethinking their role in understanding workplace behavior and practice. SIOP organizational Frontiers series. New York, NY: Routledge.

Super, D. E. (1964). A developmental approach to vocational guidance: Recent theory and results. Vocational Guidance Quartely, 13 (1), $1-10$. Doi.org/10.10002/j.2164585X.1964.tb00688.x
Super, D. E. (1990). A life-span, life-space approach to career development. In D. Brown e L. Brooks (Eds.), Career choice and development. Applying contemporary theories to practice (2nd edition, pp. 197-261). San Francisco, CA: Jossey-Bass Publishers.

Super, D. E. (1995). Models of career development. In J. F. Marques \& M. Rafael (Eds.), Conferência Internacional AIOSP. Actas. Tema geral: Serviços de Orientação para os anos 90 (pp.47-66). Lisboa, Portugal.

Super, D. E., Savickas, M. L., \& Super, C. M. (1996). The life-span, life-space approach to careers. In D. Brown \& L. Brooks and al (Eds.), Career choice and development (3rd edition, pp. 121-178). San Francisco, CA: Jossey-Bass Publishers.

Teixeira, M. O., Barros, A., \& Janeiro, I. (2010). Análise transcultural dos interesses, resultados do SDS em amostras de Portugal e do Brasil. Actas do VII Simpósio Nacional de Investigação em Psicologia. Universidade do Minho, 4 a 6 de Fevereiro, 3796-3805.

Teixeira, M. O., \& Laranjeira, M. (2018). Interesses e perceções de competência na infância: Um estudo exploratório no contexto português. Revista Iberoamericana de Diagnóstico y Evaluación - e Avaliação Psicológica, 47(2), pp. 37-52 http://dx.doi.org/10.21865/RIDEP47.2.03

Van Esbroeck, R. (2008). Career guidance in a global world. In J. A. Athanasou \& R. V. Esbroeck (Eds.), International Handbook of Career Guidance (pp. 23-44). New York: Springer.

Wang, M., \& Wanberg, C. R. (2017). 100 years of applied psychology research on individual careers: From career management to retirement. Journal of Applied Psychology, $102 \quad$ (3), 546-563. http://dx.doi.org/10.1037/apl0000143 